\title{
Bronchial Epithelial Cells from Cystic Fibrosis Patients Express a Specific Long Non-coding RNA Signature upon Pseudomonas aeruginosa Infection
}

\author{
Viviane Balloy ${ }^{1}$, Remya Koshy ${ }^{2}$, Lea Perra ${ }^{1}$, Harriet Corvol ${ }^{1,3}$, Michel Chignard ${ }^{1}$, \\ Loïc Guillot ${ }^{* t}$ and Vinod Scaria ${ }^{2,4 *}$ \\ ${ }^{1}$ Sorbonne Universités, UPMC Univ Paris 06, INSERM, Centre de Recherche Saint-Antoine (CRSA), Paris, France, ${ }^{2}$ GN \\ Ramachandran Knowledge Center for Genome Informatics, CSIR Institute of Genomics and Integrative Biology, Delhi, India, \\ ${ }^{3}$ Pneumologie Pédiatrique, AP-HP, Hôpital Trousseau, Paris, France, ${ }^{4}$ CSIR Institute of Genomics and Integrative Biology, \\ Academy of Scientific and Innovative Research, Delhi, India
}

OPEN ACCESS

Edited by:

Silvia Mercedes Uriarte University of Louisville, United States

Reviewed by:

Gregory Anderson, Indiana University - Purdue University Indianapolis, United States

Gill Diamond,

University of Florida, United States

*Correspondence: Loïc Guillot

loic.guillot@inserm.fr Vinod Scaria

vinods@igib.in

${ }^{\dagger}$ These authors have contributed equally to this work

Received: 02 February 2017 Accepted: 11 May 2017 Published: 29 May 2017

Citation:

Balloy V, Koshy R, Perra L, Corvol H, Chignard M, Guillot $L$ and Scaria V (2017) Bronchial Epithelial Cells from Cystic Fibrosis Patients Express a Specific Long Non-coding RNA Signature upon Pseudomonas aeruginosa Infection.

Front. Cell. Infect. Microbiol. 7:218. doi: 10.3389/fcimb.2017.00218
Pseudomonas aeruginosa $(\mathrm{Pa})$ is the leading cause of chronic lung infection in Cystic Fibrosis (CF) patients. It is well recognized that CF epithelial cells fail to develop an appropriate response to infection, allowing bacterial colonization and a chronic inflammatory response. Since long non-coding RNAs (IncRNAs), are known to play a key role in regulating mammalian innate immune response, we hypothesized that CF cells exposed to $\mathrm{Pa}$ could express a specific IncRNA signature responsible of the maladaptative CF response. We analyzed transcriptomic datasets to compare the expression profiles of IncRNAs in primary CF and non-CF epithelial cells infected with $\mathrm{Pa}$ at $0,2,4$, and $6 \mathrm{~h}$ of infection. Our analysis identified temporal expression signatures of $25,73,15$, and 26 IncRNA transcripts differentially expressed at $0,2,4$, and 6 h post-infection respectively, between CF and non-CF cells. In addition, we identified profiles specific to CF and non-CF cells. The differential expression of two candidate IncRNAs were independently validated using real-time PCR. We identified a specific CF signature of IncRNA expression in a context of $P a$ infection that could potentially play a role in the maladaptive immune response of CF patients.

Keywords: cystic fibrosis, lung, epithelium, Pseudomonas aeruginosa, IncRNA

\section{INTRODUCTION}

Cystic fibrosis (CF) is an autosomal recessive genetic disorder affecting one in 3,500 live births, in Caucasian populations. This disease is due to mutations in the cystic fibrosis transmembrane conductance regulator (CFTR) gene encoding the transporter channel for chloride and bicarbonate ions in epithelial cells. In airways, a deficient CFTR results in excessive secretion of abnormally thick and viscous mucus with impairment of innate immune host defenses. This results in chronic infection and inflammation, which together acts to further deteriorate the lung function (Palmer and Whiteley, 2015). Pseudomonas aeruginosa $(\mathrm{Pa})$ is the leading cause of chronic lung infections and is responsible for significant morbidity and mortality in CF patients. Chronic $\mathrm{Pa}$ infection is characterized by repeated episodes of pulmonary exacerbations, during which lung function declines abruptly (Elborn, 2016). 
Innate immunity system provides immediate defense against microbial invasion by recruiting immune cells and driving inflammation signals to sites of infection. Response of pulmonary epithelial cells to external stimuli requires tight regulation of genes involved in the innate immune response. This fine-tuned regulation, at the transcriptional and post-transcriptional levels, is necessary to prevent detrimental effects of uncontrolled cell activation. In a previous study, we compared transcriptomes of $\mathrm{CF}$ and non-CF bronchial epithelial cells during $\mathrm{Pa}$ infection (Balloy et al., 2015). We observed different profiles with genes up- and down-regulated in CF compared to non-CF cells that could illuminate the inflammatory response or be involved in the fighting of pathogens as well as in the decreased protection of tissue integrity.

Non-coding RNAs (ncRNAs) have recently emerged as important regulators of gene expression. Long ncRNAs (lncRNAs) larger than 200 nucleotides, represent the largest class of the ncRNA molecules. Although the current strongest evidence supports their role in cancer (Tsai et al., 2011), lncRNAs play key roles in regulating mammalian innate immune responses as well (Sigdel et al., 2015). Depending on their localization, they act on gene expression through interactions with DNA, RNA or proteins (Rinn and Chang, 2012) through various mechanisms including chromatin remodeling, epigenetic regulation, transcription, mRNA splicing, RNA decay and enhancer functions (Li et al., 2016).

It has been previously shown that lncRNA expression profile is altered in inflammatory lung diseases such as chronic obstructive pulmonary diseases (COPD), asthma and acute lung injury (ALI) (Xie and Liu, 2015). In CF, the description and the role of lncRNAs is beginning to be unraveled. However, CFTR expression and chloride ion function was recently shown to be regulated by lncRNA (McKiernan et al., 2014). A total of 1,063 lncRNAs with differential expression were identified from CF bronchial brush sampling compared to non-CF (Saayman et al., 2016). In particular, XIST and TLR8-AS1 were confirmed to be respectively up- and down-regulated in CF compared to nonCF samples. However, in this study, the influence of infection was not addressed, although there are increased evidences of lncRNA involvement in infectious diseases (Scaria and Pasha, 2012). Reports have revealed roles for lncRNAs as regulators of antimicrobial functions such as the lncRNA NEAT1 which directly regulates HIV replication (Zhang et al., 2013) or the lncRNA NRAV that functions as a negative regulator of the host antiviral immunity by repressing the expression of IFNstimulated genes through strict control of the transcription rate (Ouyang et al., 2014).

We therefore investigated the differential expression of lncRNAs in CF and non-CF epithelial cells during infection by $\mathrm{Pa}$ that may partly mimic and possibly explain the differential mRNA profiles between the two cell types observed previously (Balloy et al., 2015). In the present study, we re-analyzed the RNA-seq data with this perspective and evaluated the expression profiles of lncRNAs in infected $\mathrm{CF}$ and non$\mathrm{CF}$ epithelial cells at the aim of identifying a specific CF signature for lncRNA expression in the presence of $\mathrm{Pa}$ infection.

\section{METHODS}

\section{Dataset}

Raw datasets publicly available (European Nucleotide Archive (ENA), primary accession number PRJEB9292) were from a transcriptional analysis designed to compare the molecular determinants of $\mathrm{CF}$ and non-CF epithelial cells to $\mathrm{Pa}$ infection. The PAK strain of $\mathrm{Pa}$ (given by Stephen Lowry) is relevant for pulmonary infections in the context of CF Like most of clinical strains responsible for CF infection, it expresses a full complement of virulence factors, including pili, flagella, type II secreted enzymes, type III secreted exo-enzymes S, T, and $\mathrm{Y}$, exotoxin A, elastase and phospholipase. The data were generated from human bronchial epithelial cells from four CF patients homozygous for the p.F508del mutation and four healthy donors (non-CF), each infected with $\mathrm{Pa}$ at 0.25 Multiplicity of Infection (MOI) for $0,2,4$, and $6 \mathrm{~h}$. The data with an average of over 150 million reads with each data set were previously published (Balloy et al., 2015).

\section{Reads Alignment, Mapping, and Assembly}

Read quality was checked with FastQC program, a quality control tool for high throughput sequence data (http://www. bioinformatics.babraham.ac.uk/projects/fastqc/) and reads were further aligned to the hg38 build of the human genome (UCSC Genome Browser). The splice-aware aligner Tophat (Mortazavi et al., 2008) (v2.0.11) which is integrated with Bowtie2, a fast and sensitive gapped read aligner, was used for aligning the sequence reads. One dataset was excluded from further analysis after observing only less than $85 \%$ of read alignment. Datasets, raw reads and the proportion of aligned reads are summarized in Supplementary Table 1. The expression levels across the transcripts and genes were based on GENCODE v23 (http://www.GENCODEgenes.org/). Briefly, this annotation set comprised of a total of 198,619 transcripts in the human genome, including 79,795 protein-coding and 118,824 non-coding transcripts, among which 27,817 lncRNAs. The expression levels were computed as fragments per kilobase of exon per million (FPKM) units and the differential expression between the different sets were computed using cuffdiff.

\section{Identifying Potential Differentially Expressed IncRNA Transcripts}

We used a nominal cutoff of 1 FPKM to filter genes and transcripts. The Supplementary Table 2 lists out the number of genes and transcripts covered by each sample within the range of FPKM $\geq 1,5$, and 10 . The differentially expressed genes and transcripts were filtered using stringent cutoffs. We considered lncRNAs with FPKM $\geq 1$ with a log2-fold change $\leq-1$ or $\geq 1$ and $p \leq 0.05$ as differentially expressed.

We performed two orthologous analyses across datasets. We first determined genes and transcripts which were differentially up- or down-regulated at each infection time points between CF and non-CF epithelial cells. Then, we examined the lncRNA transcripts differentially expressed between the $0 \mathrm{~h}$ time point and 2, 4, and $6 \mathrm{~h}$ post-infection in both CF and non-CF groups analyzed separately. From this, we sorted out the differentially 
expressed lncRNA transcripts which were found only in CF or in non-CF cells. The overlapped transcripts of the first set comprising the differentially expressed lncRNA transcripts of $\mathrm{CF} /$ non-CF analysis and second set comprising the differentially expressed transcripts at 2, 4, and $6 \mathrm{~h}$ post-infection, identified only in CF cells, and gave us the potential lncRNA transcripts with potential for association with $\mathrm{Pa}$ infection in CF epithelial cells.

\section{Validation of Expression Level Using qRT-PCR}

Quantitative PCR was performed using an ABI StepOnePlus ${ }^{\mathrm{TM}}$ (Applied Biosystems, Carlsbad, CA, USA) and TaqMan technology. TaqMan probes used (Applied Biosystems) were XIST (Hs01079824), MEG9 (Hs01593046), BLACAT1 (Hs03839366), and GAPDH (Hs02758991). Relative quantifications of the lncRNA level were carried out using the $2^{-\triangle \Delta \mathrm{Ct}}$ method, and normalized with respect to GAPDH and for the expression levels to respective non-infected cells (T0). Each sample was assessed in triplicates to ensure experiment quality.

\section{Statistical Analysis}

Data were described as mean \pm SEM. Between-group differences were tested using the paired $t$-test. Values of p lower than 0.05 were considered significant; in the figures, statistically significant differences with $p<0.05\left(^{*}\right), p<0.01\left(^{* *}\right)$, and $p<0.001\left(^{* * *}\right)$ are indicated.

\section{Correlation Analysis of Gene Expression Data}

Correlation analysis was carried out using Pearson's algorithm with the help of $\mathrm{R}$ package; rcorr. We observed the mRNA transcripts positively or negatively correlated with the overlapped lncRNAs from two analyses. Transcripts with an empirical r-score greater than 0.85 were considered to be positively regulated whereas transcripts with $r$-score less than -0.85 were considered to be negatively regulated and $p$-value less than 0.01 was considered as significant. We additionally performed enrichment analysis for the protein coding genes coregulated with the lncRNAs using DAVID functional annotation tool.

\section{RESULTS}

\section{Identification of Temporal Infection Signatures from RNA-Seq Data}

For identifying differentially expressed genes and transcripts in CF compared to non-CF epithelial cells, we used cuffdiff and the reference gene and transcript annotations from the GENCODE version 23 for guiding the assembly. The cuffdiff output provided FPKM values, log2-fold change value of CF upon non-CF for every time points studied. The numbers of genes differentially expressed at $0,2,4$, and $6 \mathrm{~h}$ are found to be 297, 958, 281, and 439 respectively and a total of 336, 958, 336, and 518 transcripts respectively (Table 1). Their FPKM values and fold changes are summarized in Supplementary Table 3. The lncRNA annotation files were separately retrieved from the GENCODE database to extract all the significantly differentially expressed lncRNA transcripts. We identified a total of 25, 73, 15, and 26 differentially expressed lncRNAs, corresponding to the $0,2,4$, and $6 \mathrm{~h}$ time points. The unique number of lncRNA transcripts differentially expressed throughout the dataset was found to be 108. These latter are shown in a Venn diagram (Figure 1) to visualize shared lncRNAs differentially expressed between CF

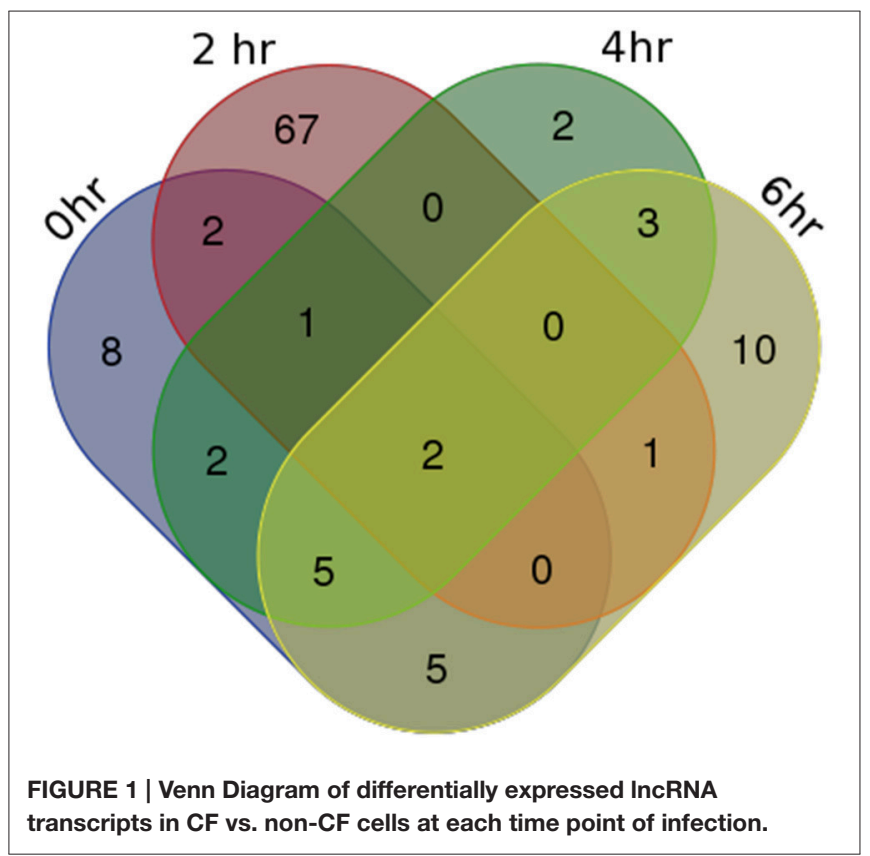

TABLE 1 | Summary of genes and transcripts differentially expressed in CF vs. non-CF cells at each time point of infection.

\begin{tabular}{|c|c|c|c|c|}
\hline Differentially expressed genes/transcripts & $\mathrm{O} \mathrm{h}$ (CF/non-CF) & $2 \mathrm{~h}$ (CF/non-CF) & $4 \mathrm{~h}$ (CF/non-CF) & $6 \mathrm{~h}$ (CF/non-CF) \\
\hline Transcripts & 336 & 958 & 336 & 518 \\
\hline IncRNA genes & 21 & 73 & 15 & 22 \\
\hline Unique IncRNA transcripts & \multicolumn{4}{|c|}{108} \\
\hline
\end{tabular}


and non-CF infected cells. Out of the 108 lncRNA transcripts, a total of $12 \operatorname{lncRNAs}$ are differentially expressed in at least 2 time points. Table 2 lists these lncRNAs, their cognate gene locus and functions as annotated from literature. Three of the 12 lncRNAs have been previously shown to be expressed in the human lung. The lncRNA RP11-44F21.5 has been previously suggested to be down-regulated in squamous cell carcinoma of the lung in the following analysis: https:/genevisible.com/perturbations/ HS/Gene\%20Symbol/RP11-44F21.5. The two other lncRNAs,
LINC00704 and LINC00992, have been shown to be expressed in lungs, kidneys and salivary glands (Stelzer et al., 2016).

\section{Highlighting Distinct Specific Infection Signatures in Non-CF and CF Cells}

To identify the lncRNA transcripts specific of $\mathrm{Pa}$ infection, we used cuffdiff to compare the expression values for each time point against $0 \mathrm{~h}$ for $\mathrm{CF}$ and non-CF datasets separately. We retrieved the differentially expressed transcripts using

TABLE 2 | Summary of IncRNA genes differentially expressed in CF vs. non-CF cells for at least 2 of the time points of infection.

\begin{tabular}{llll}
\hline $\begin{array}{l}\text { Conditions differentially } \\
\text { expressed }\end{array}$ & IncRNA & Ensembl ID & Function
\end{tabular}

$\begin{array}{llll}0 & 2 & 4 & 6\end{array}$

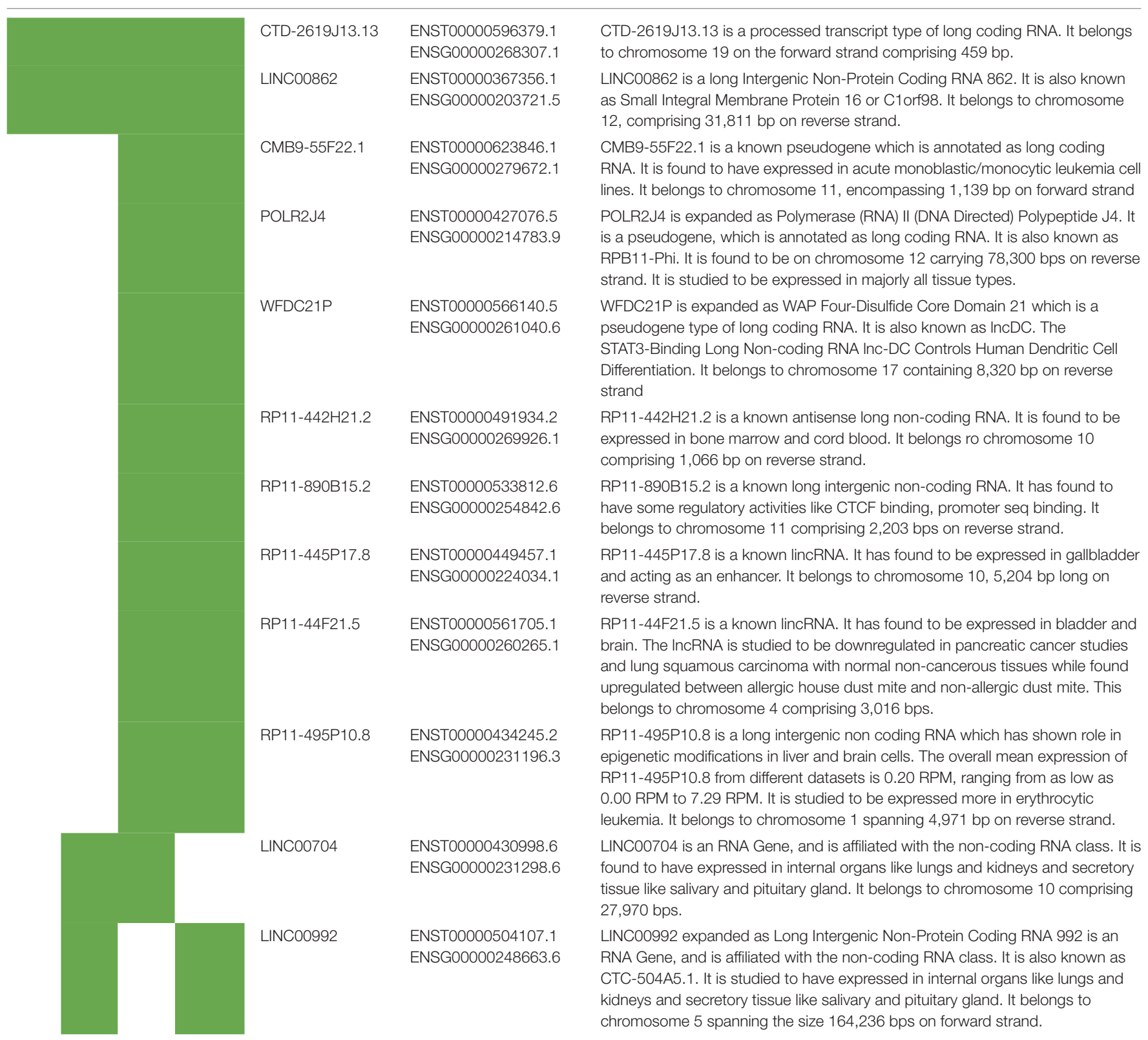


TABLE 3 | Genes and IncRNAs differentially expressed in CF and non-CF cells at different time points of infection compared to the non-infected status $(\mathrm{O} h)$.

\begin{tabular}{|c|c|c|c|c|c|c|}
\hline \multirow{2}{*}{$\begin{array}{l}\text { Differentially expressed } \\
\text { genes/transcripts }\end{array}$} & \multicolumn{2}{|c|}{$2 \mathrm{~h}$ vs. $0 \mathrm{~h}$} & \multicolumn{2}{|c|}{$4 \mathrm{~h}$ vs. $0 \mathrm{~h}$} & \multicolumn{2}{|c|}{$6 \mathrm{~h}$ vs. $0 \mathrm{~h}$} \\
\hline & $\mathrm{CF}$ & Non-CF & CF & Non-CF & CF & Non-CF \\
\hline Genes & 72 & 587 & 99 & 75 & 272 & 140 \\
\hline Transcripts & 72 & 603 & 102 & 76 & 305 & 146 \\
\hline IncRNA transcripts & 2 & 53 & 1 & 7 & 17 & 5 \\
\hline IncRNA Genes & 2 & 53 & 1 & 7 & 17 & 5 \\
\hline Unique IncRNA transcripts & & & & 80 & & \\
\hline Unique IncRNA genes & & & & 79 & & \\
\hline
\end{tabular}

the same filters as mentioned previously. The total number of genes or transcripts, differentially expressed were found comparatively less in CF than in non-CF samples at $2 \mathrm{~h}$ vs. $0 \mathrm{~h}$ (Table 3). We further analyzed the common lncRNA transcripts differentially expressed at each time point in $\mathrm{CF}$ and non$\mathrm{CF}$ datasets. Except for 2 up-regulated lncRNAs at $6 \mathrm{~h}$ vs. 0 $\mathrm{h}$, we obtained two distinct profiles of lncRNAs as none of lncRNAs differentially expressed during infection in non-CF are common to those differentially expressed in CF cells (Figure 2). The FPKM values and fold changes of the up- and downregulated transcripts are summarized in Supplementary Tables 4, 5 , respectively.

For finding out the lncRNA signature specific to infected $\mathrm{CF}$ cells in the one hand and to non-CF cells in the other hand, we summarized overlaps between differentially expressed (both up and down) transcripts in the CF sets (Figure 3A) and in the non-CF sets (Figure 3B) respectively for each of the respective time points against non-infected time point $(0$ h). We found that $62 \operatorname{lncRNA}$ transcripts (62 up-regulated) are specifically regulated in $\mathrm{Pa}$ infected non-CF epithelial cells, whereas 17 lncRNA transcripts (2 up- and 15 downregulated) are specific of the $\mathrm{CF}$ epithelial cell response to the infection. The FPKM values and fold changes of the upregulated transcripts in non-CF cells and the up- and downregulated in CF cells are summarized in Supplementary Tables 6-8, respectively.

Among the lncRNA transcripts specific signature of infected CF cells, we selected two of them already studied in the literature, MEG9 and BLACAT1, for validation of their expression by qPCR. Thus, the pattern of expression found by in silico analysis for MEG9 and BLACAT1 was confirmed with a significant down regulation at 2 and $6 \mathrm{~h}$ post-infection, respectively (Figure 4). One lncRNA transcript, XIST, identified in the infected non-CF signature and already described by Greene's team comparing $\mathrm{CF}$ and non-CF samples, was measured by qPCR. Validation of its expression was not as clear as for MEG9 and BLACAT1. Indeed, we observed varying levels of expression between epithelial cells derived from male and female, independently of CFTR genotype, i.e., very low or no expression in male cells (Ct around 36 or undetermined) whereas high expression in female cells (Ct around 18.5) (data not shown).

\section{Proteins Coding Transcripts Correlated with BLACAT1 and MEG9 Expression}

The Gene Ontology analysis did not reveal protein-coding genes significantly correlated with BLACAT1 expression, but it identified protein coding transcripts positively and negatively correlated with lncRNA MEG9. Among the genes positively correlated, we observed $62.3 \%$ of proteins engaged in protein binding, $18.5 \%$ in positive regulation of cellular process and around $10 \%$ in extracellular matrix. The genes negatively correlated (17.4\%) with lncRNA MEG9 were involved in structural components of ribosome (Table 4).

\section{DISCUSSION}

The airway epithelium is the first site in contact with inhaled pathogens. Its response to bacterial invasions activates innate immune mechanisms through the involvement of various receptors. In CF patients, this normal process is disturbed leading to a decrease of the bacterial clearance resulting in chronic infection in lungs.

Over the years, lncRNAs have been recognized as major regulators of multiple cellular processes among which the regulation of immune effectors. They can interact with transcription factors which is the case of the IncRNA NRON that inhibits the translocation of NFAT in the nucleus by sequestering it in the cytosol and so negatively regulating T-cell activation (Willingham et al., 2005). Three lncRNAs (murine NeST, human THRIL, and NEAT1) have been previously shown to regulate the innate immune response by modulating the transcription of cytokines such as IFN- $\gamma$, TNF- $\alpha$, and IL-8 (Cullen, 2013; Gomez et al., 2013; Imamura et al., 2014; Li et al., 2014). It is also known that toll-like receptor (TLR) signaling can activate endogenous feedback-regulation networks to limit the potentially damaging effects of an excessive inflammation. This is the case with the lncRNA THRIL which helps in down-regulation to restrain TLR-induced gene activation (Li et al., 2014).

LncRNA expression is tissue-specific indicating a tight regulation that could be affected by $\mathrm{Pa}$ infection in CF patients. For this reason, we decided to explore the expression of lncRNAs in the infectious context of respiratory epithelial cells that are key cells involved in CF. CF vs. non-CF infected cells study brought 108 unique lncRNAs as differentially up- or down-regulated with 12 lncRNAs which are expressed in at least two time points. Among them, the lncRNAs, LINC00862, and CTD-2619J13, are differentially expressed at all four time points in spite of bacterial invasion. As such, they could be two potential signatures for CF. Most of the 108 unique lncRNAs was differentially expressed at $2 \mathrm{~h}$, almost immediately after bacterial attack. According to Ensembl and lncRNAdb, some of these differentially expressed lncRNAs are involved in regulatory activities namely CTCF binding, promoter sequence binding transcripts and few were found differentially expressed in different tissues such as in lungs and in erythrocytes.

To pinpoint a specific signature related to infection in the CF vs. non-CF context, we took in account lncRNA transcripts specific to $\mathrm{Pa}$ infection expressed only in $\mathrm{CF}$ in one hand and in 

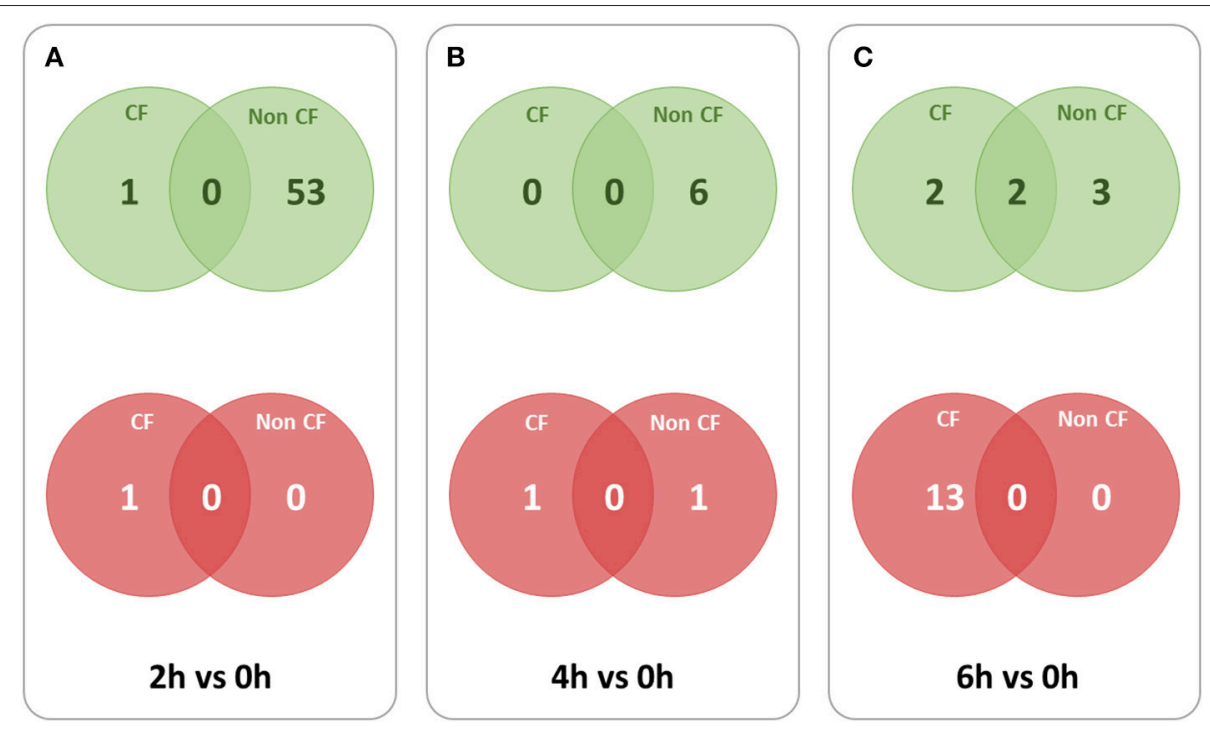

FIGURE 2 | Overlaps between IncRNA transcripts differentially expressed at different time points in CF and non-CF datasets. Red circles represent down-regulated transcripts and green circles represent up-regulated transcripts. (A) $2 \mathrm{~h}$ in comparison with $0 \mathrm{~h}$ time point, (B) $4 \mathrm{~h}$ in comparison with $0 \mathrm{~h}$ time point, (C) $6 \mathrm{~h}$ in comparison with $0 \mathrm{~h}$ time point.
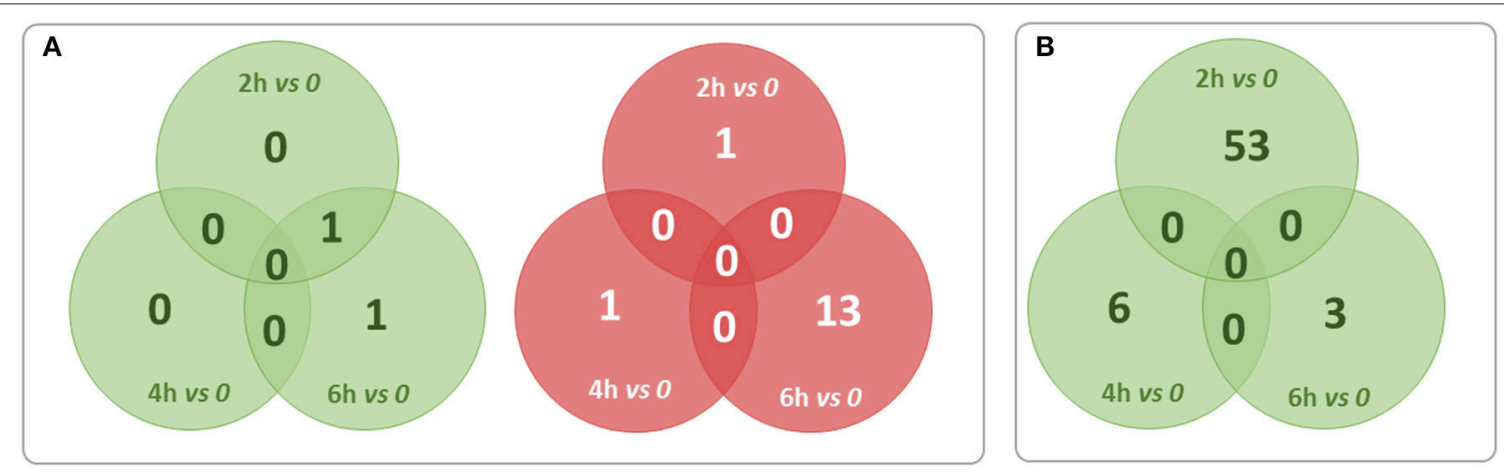

FIGURE 3 | Summary of overlaps between differentially expressed transcripts only in the CF set (A) and only in non-CF set (B) at each of the respective time points against non-infected time point $(0 \mathrm{~h})$. Red circles represent down-regulated transcripts and green circles represent up-regulated transcripts. No transcripts were found down-regulated exclusively in non-CF set.

non-CF cells in other hand from each time point (i.e., 2, 4, and 6 h) and compared with their expression in uninfected cells.

Numbers of lncRNAs differentially expressed for CF and nonCF cells are totally different with 17 and 62 lncRNAs, respectively. Among the lncRNAs belonging to the specific signature of $\mathrm{Pa}$ infected non-CF cells; we observed a well annotated lncRNA, XIST. As described by Greene's team ${ }^{9}$, we detected the IncRNA XIST more expressed in CF than in non-CF cells at the noninfected status (Fold change $>4$ ). Moreover, we observed that XIST expression is up-regulated in non-CF cells during infection at 4 and $6 \mathrm{~h}(\mathrm{FC}>2)$. Nonetheless, the validation of XIST by qPCR does not fit with the in silico analysis. Indeed, we observed differences of expression between cells from male and female donors but no differences of expression between CF and non-CF epithelial cells. This result is consistent with the literature as XIST is a lncRNA required for the establishment of
$\mathrm{X}$-chromosome silencing in placental mammals. X inactivation is an early developmental process in mammalian females that transcriptionally silences one of the pair of $\mathrm{X}$ chromosomes, thus providing dosage equivalence between males and females (Furlan and Rougeulle, 2016). Due to the differences in the gender of donors, taken individually, values did not show the variations averaged in silico analysis. Other transcripts, ENST00000452120.6 and ENST00000398461.5 were found in the CF cells specific signatures. These transcripts are annotated as MEG3 (Maternally Expressed 3) which is a maternally imprinted gene with all spliced transcripts annotated as lncRNAs and is a well-known lncRNA tumor suppressor. MEG3 has been previously reported to regulate the TGF- $\beta$ pathway genes by forming RNA-DNA triplex structures (Mondal et al., 2015). This pathway is frequently regulated during tissue injury and repair and the up-regulation of TGF- $\beta$ is involved in lung fibrogenic 

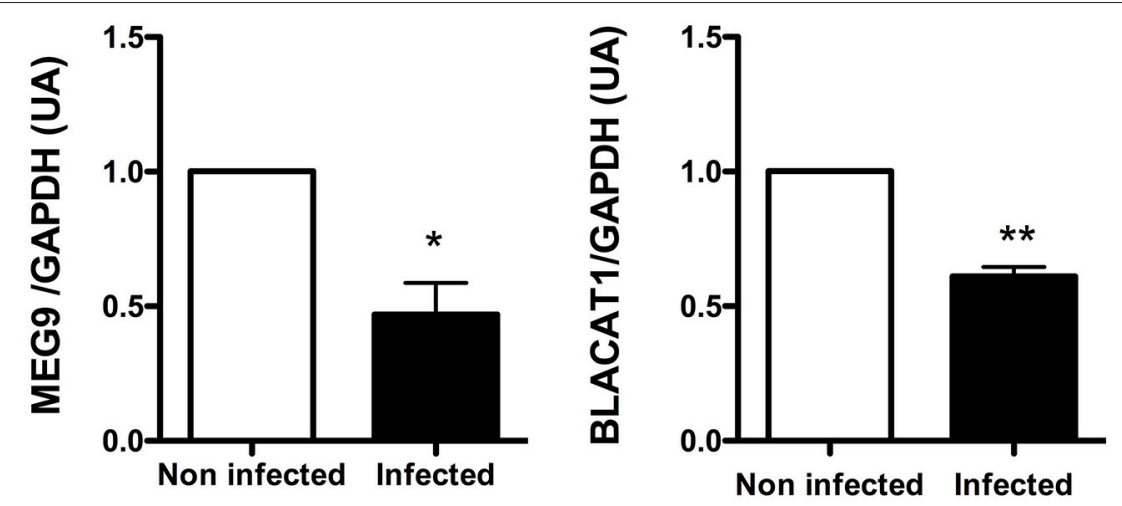

FIGURE 4 | Validation by qRT-PCR of BLACAT1 and MEG9 expressions in bronchial primary epithelial CF cells infected with Pa. RNA were purified in CF cells collected from 4 different patients and infected with Pa. LncRNA levels were measured at 0 (white bar) and 2 h (black bar) post-infection for MEG9 and 0 (white bar) and $6 \mathrm{~h}$ (black bar) post-infection for BLACAT1. The statistical analysis consisted in a paired $t$-test. ${ }^{*} P<0.05$ and ${ }^{* \star} P<0.001$.

TABLE 4 | DAVID's GO terms for protein coding transcripts positively and negatively correlated with the IncRNA MEG9.

\begin{tabular}{|c|c|c|c|c|c|}
\hline Category & GO term & Count & Percentage & $P$-value & Benjamini corrected $p$-value \\
\hline \multicolumn{6}{|c|}{ GENES POSITIVELY CORRELATED IN EXPRESSION } \\
\hline \multirow[t]{2}{*}{ Biological process } & Regulation of localization & 19 & 9.2 & $3.0 \mathrm{E}-4$ & 4.6E-2 \\
\hline & Positive regulation of cellular process & 38 & 18.5 & 7.0E-4 & 5.30E-02 \\
\hline \multirow[t]{2}{*}{ Cellular components } & Extracellular matrix part & 10 & 4.8 & 1.3E-5 & 4.4E-4 \\
\hline & Extracellular matrix & 14 & 6.8 & 3.1E-4 & $5.3 E-3$ \\
\hline Molecular function & Protein binding & 129 & 62.3 & $1.60 \mathrm{E}-4$ & 7.80E-3 \\
\hline \multicolumn{6}{|c|}{ GENES NEGATIVELY CORRELATED IN EXPRESSION } \\
\hline Molecular function & Structural constituent of ribosome & 4 & 17.4 & 1.9E-3 & $3.10 \mathrm{E}-2$ \\
\hline
\end{tabular}

diseases. The expression of the lncRNA MEG3 in signature could be associated with the absence of injury after invasion of $\mathrm{Pa}$ in non-CF. A recent study in mice characterized MEG3 as a novel pulmonary inflammatory regulator of bacterial infection through miR-138 (Li and Wu, 2016).

The highlighting of a specific signature in CF epithelial cells in response to $\mathrm{Pa}$ infection revealed 17 lncRNA transcripts. Among them, 4 lncRNA transcripts MEG9, BLACAT1, RP11477I4.4, and RP11-1334A24.5 overlapped with the 108 unique lncRNAs which are differentially expressed between CF and non-CF cells. RT-PCR gave a similar pattern of expression of MEG9 and BLACAT1 as from in silico analysis and validate the different signatures obtained with the computational analysis. ENST00000626538.1, otherwise known as lncRNA BLACAT1 (Bladder Cancer Associated Transcript 1), was found down-regulated in CF patients. Until now, BLACAT1, firstly characterized in bladder cancer, was also described to be involved in the development of gastric cancer. It has been shown that its depletion decreased the gastric cancer cell proliferation, motility and invasion ( $\mathrm{Hu}$ et al., 2015). Since shedding and abnormal repair are characteristics of CF epithelial cells, it would be interesting to study whether their altered expression of BLACAT1 influences their repair, proliferation and migration. In our study, analysis did not reveal protein-coding gene significantly correlated with BLACAT1 expression. However, as shown previously BLACAT1 could interact directly with other molecular partners than transcripts such as the proteins of the polycomb repressive complex 2 (PRC2) (He et al., 2013).

Concerning MEG9 (Maternally Expressed 9), a lncRNA belonging to the MEG family, it has been described to be up-regulated upon hypoxia in human endothelial cells and in a model of mouse ischemia (Voellenkle et al., 2016). In our study, MEG9 was found dramatically down-regulated $2 \mathrm{~h}$ postinfection compared to the non-infected status $(0 \mathrm{~h})$ in $\mathrm{CF}$ cells (Supplementary Table 8). From our correlation analysis of gene expression, MEG9 was found co-regulated positively with 226 and negatively with 25 protein coding genes. Gene ontology analysis showed that MEG9 was found positively coregulated mostly with proteins engaged in protein binding (62.3\%), in positive regulation of cellular process (18.5\%) and few of them in extracellular matrix (Table 4), all processes involved in inflammation of the lung in CF (Cohen-Cymberknoh et al., 2013).

Our study showed that $\mathrm{Pa}$ infection altered the expression of lncRNAs in CF respiratory epithelial cells which could have a potential role in the innate immune system and thus could contribute to the maladaptive immune response observed in patients. We have experimentally validated the two potential transcripts namely MEG9 and BLACAT1 expression, which 
were found differentially expressed in both $\mathrm{CF} /$ non-CF and infected/non-infected analyses. Further investigations either in vitro (respiratory epithelial cells in culture) or in vivo (study of conserved LncRNA (MEGs) in mice model of pulmonary infection) are necessary to understand the role of these lncRNAs in the innate immune response. The present study may contribute to provide further insight into the biological functions and molecular mechanisms of lncRNAs regulation in CF in which $\mathrm{Pa}$ infection plays a crucial role.

\section{AUTHOR CONTRIBUTIONS}

Conceived and designed the experiments: LG, VB, and MC. Performed the biological experiments: LG, VB. Analyzed the data: RK, LG, VB, and VS. Wrote the paper: RK, VB, LG, MC, LP, HC, and VS.

\section{REFERENCES}

Balloy, V., Varet, H., Dillies, M.-A., Proux, C., Jagla, B., Coppée, J.-Y., et al. (2015). Normal and cystic fibrosis human bronchial epithelial cells infected with Pseudomonas aeruginosa exhibit distinct gene activation patterns. PLOS ONE 10:e0140979. doi: 10.1371/journal.pone. 0140979

Cohen-Cymberknoh, M., Kerem, E., Ferkol, T., and Elizur, A. (2013). Airway inflammation in cystic fibrosis: molecular mechanisms and clinical implications. Thorax 68, 1157-1162. doi: 10.1136/thoraxjnl-2013203204

Cullen, B. R. (2013). Making a NeST for a persistent virus. Cell Host Microbe 13, 241-242. doi: 10.1016/j.chom.2013.02.016

Elborn, J. S. (2016). Cystic Fi Brosis. Available online at: www.thelancet.com, 388.

Furlan, G., and Rougeulle, C. (2016). Function and evolution of the long noncoding RNA circuitry orchestrating X-chromosome inactivation in mammals. Wiley Interdiscip. Rev. RNA 7, 702-722. doi: 10.1002/ wrna.1359

Gomez, J. A., Wapinski, O. L., Yang, Y. W., Bureau, J.-F., Gopinath, S., Monack, D. M., et al. (2013). The NeST long ncRNA controls microbial susceptibility and epigenetic activation of the interferon- $\gamma$ locus. Cell 152, 743-754. doi: 10.1016/j.cell.2013.01.015

He, W., Cai, Q., Sun, F., Zhong, G., Wang, P., Liu, H., et al. (2013). lincUBC1 physically associates with polycomb repressive complex 2 (PRC2) and acts as a negative prognostic factor for lymph node metastasis and survival in bladder cancer. Biochim. Biophys. Acta 1832, 1528-1537. doi: 10.1016/j.bbadis.2013.05.010

Hu, Y., Pan, J., Wang, Y., Li, L., and Huang, Y. (2015). Long noncoding RNA linc$\mathrm{UBC} 1$ is negative prognostic factor and exhibits tumor pro-oncogenic activity in gastric cancer. Int. J. Clin. Exp. Pathol. 8, 594-600.

Imamura, K., Imamachi, N., Akizuki, G., Kumakura, M., Kawaguchi, A., Nagata, K., et al. (2014). Long noncoding RNA NEAT1-dependent SFPQ relocation from promoter region to paraspeckle mediates IL8 expression upon immune stimuli. Mol. Cell 53, 393-406. doi: 10.1016/j.molcel.2014. 01.009

Li, R., and Wu, M. (2016). Long noncoding RNA MEG3-4 regulates IL-1 $\beta$ during pulmonary bacterial infection by repressing microRNA-138. J. Immunol. 196(1 Suppl.), 127.9.

Li, R., Zhu, H., and Luo, Y. (2016). Understanding the functions of long noncoding RNAs through their higher-order structures. Int. J. Mol. Sci. 17:702. doi: 10.3390/ijms17050702

Li, Z., Chao, T.-C., Chang, K.-Y., Lin, N., Patil, V. S., Shimizu, C., et al. (2014). The long noncoding RNA THRIL regulates TNF expression through its interaction with hnRNPL. Proc. Natl. Acad. Sci. U.S.A. 111, 1002-1007. doi: $10.1073 /$ pnas. 1313768111

\section{ACKNOWLEDGMENTS}

This work was supported by a grant from two French nonprofit cystic fibrosis organizations, Vaincre la mucoviscidose and Association Gregory Lemarchal. The analysis was funded by CSIR, India through Grant BSC0123(GENCODE-C). The funders had no role in study design, data collection and analysis, decision to publish, or preparation of the manuscript. Authors also acknowledge Dr. Srinivasan Ramachandran for editorial help.

\section{SUPPLEMENTARY MATERIAL}

The Supplementary Material for this article can be found online at: http://journal.frontiersin.org/article/10.3389/fcimb. 2017.00218/full\#supplementary-material

McKiernan, P. J., Molloy, K., Cryan, S. A., McElvaney, N. G., and Greene, C. M. (2014). Long noncoding RNA are aberrantly expressed in vivo in the cystic fibrosis bronchial epithelium. Int. J. Biochem. Cell Biol. 52, 184-191. doi: 10.1016/j.biocel.2014. 02.022

Mondal, T., Subhash, S., Vaid, R., Enroth, S., Uday, S., Reinius, B., et al. (2015). MEG3 long noncoding RNA regulates the TGF- $\beta$ pathway genes through formation of RNA-DNA triplex structures. Nat. Commun. 6:7743. doi: $10.1038 /$ ncomms 8743

Mortazavi, A., Williams, B. A., McCue, K., Schaeffer, L., Wold, B., Salzberg, S. L., et al. (2008). Mapping and quantifying mammalian transcriptomes by RNA-Seq. Nat. Methods 5, 621-628. doi: 10.1038/nmeth. 1226

Ouyang, J., Zhu, X., Chen, Y., Wei, H., Chen, Q., Chi, X., et al. (2014). NRAV, a long noncoding RNA, modulates antiviral responses through suppression of interferon-stimulated gene transcription. Cell Host Microbe 16, 616-626. doi: 10.1016/j.chom.2014. 10.001

Palmer, G. C., and Whiteley, M. (2015). Metabolism and pathogenicity of Pseudomonas aeruginosa infections in the lungs of individuals with cystic fibrosis. Microbiol. Spectr. 3. doi: 10.1128/microbiolspec.MBP0003-2014

Rinn, J. L., and Chang, H. Y. (2012). Genome regulation by long noncoding RNAs. Annu. Rev. Biochem. 81, 145-166. doi: 10.1146/annurev-biochem-051410-092902

Saayman, S. M., Ackley, A., Burdach, J., Clemson, M., Gruenert, D. C., Tachikawa, K., et al. (2016). Long non-coding RNA BGas regulates the cystic fibrosis transmembrane conductance regulator. Mol. Ther. 24, 1351-1357. doi: $10.1038 / \mathrm{mt} .2016 .112$

Scaria, V., and Pasha, A. (2012). Long non-coding RNAs in infection biology. Front. Genet. 3:308. doi: 10.3389/fgene.2012.00308

Sigdel, K. R., Cheng, A., Wang, Y., Duan, L., and Zhang, Y. (2015). The emerging functions of long noncoding RNA in immune cells: autoimmune diseases. $J$. Immunol. Res. 2015, 1-9. doi: 10.1155/2015/848790

Stelzer, G., Rosen, N., Plaschkes, I., Zimmerman, S., Twik, M., Fishilevich, S., et al. (2016). The genecards suite: from gene data mining to disease genome sequence analyses. Curr. Protoc. Bioinform. 54, 1.30.1-1.30.33. doi: 10.1002/ cpbi. 5

Tsai, M.-C., Spitale, R. C., and Chang, H. Y. (2011). Long intergenic noncoding RNAs: new links in cancer progression. Cancer Res. 71, 3-7. doi: 10.1158/0008-5472.CAN-10-2483

Voellenkle, C., Garcia-Manteiga, J. M., Pedrotti, S., Perfetti, A., De Toma, I., Da Silva, D., et al. (2016). Implication of long noncoding RNAs in the endothelial cell response to hypoxia revealed by RNA-sequencing. Sci. Rep. 6:24141. doi: $10.1038 /$ srep 24141 
Willingham, A. T., Orth, A. P., Batalov, S., Peters, E. C., Wen, B. G., AzaBlanc, P., et al. (2005). A strategy for probing the function of noncoding RNAs finds a repressor of NFAT. Science 309, 1570-1573. doi: 10.1126/science. 1115901

Xie, N., and Liu, G. (2015). ncRNA-regulated immune response and its role in inflammatory lung diseases. Am. J. Physiol. Lung Cell. Mol. Physiol. 309, L1076-L1087. doi: 10.1152/ajplung.00286.2015

Zhang, Q., Chen, C.-Y., Yedavalli, V. S., and Jeang, K.-T. (2013). NEAT1 long noncoding RNA and paraspeckle bodies modulate HIV-1 posttranscriptional expression. MBio 4:e00596-12. doi: 10.1128/mbio.00 $596-12$
Conflict of Interest Statement: The authors declare that the research was conducted in the absence of any commercial or financial relationships that could be construed as a potential conflict of interest.

Copyright (c) 2017 Balloy, Koshy, Perra, Corvol, Chignard, Guillot and Scaria. This is an open-access article distributed under the terms of the Creative Commons Attribution License (CC BY). The use, distribution or reproduction in other forums is permitted, provided the original author(s) or licensor are credited and that the original publication in this journal is cited, in accordance with accepted academic practice. No use, distribution or reproduction is permitted which does not comply with these terms. 